\title{
Young Women with Breast Cancer in the United States and South Korea: Comparison of Demographics, Pathology and Management
}

\author{
Byung Ho Son ${ }^{1}$, Laura S Dominici ${ }^{2 \&}$, Fatih Aydogan ${ }^{2 *}$, Lawrence N Shulman ${ }^{3}$, \\ Sei Hyn Ahn ${ }^{1}$, Ja Young Cho ${ }^{1}$, Suzanne B Coopey ${ }^{4}$, Sung Bae Kim ${ }^{5}$, H Elise Min ${ }^{2}$, \\ Monica Valero ${ }^{2}$, Jiping Wang ${ }^{2}$, Diana Caragacianu ${ }^{2}$, Gyung-yub Gong ${ }^{6}$, Nathanael \\ D Hevelone ${ }^{2}$, Seunghee Baek ${ }^{7}$, Mehra Golshan ${ }^{2}$
}

\begin{abstract}
Background: Breast cancer diagnosed in young women may be more aggressive, with higher rates of local and distant recurrence compared to the disease in older women. Epidemiologic evidence suggests that Korean women have a lower incidence of breast cancer than women in the United States, but that they present at a younger age than their American counterparts. We sought to compare risk factors and management of young women with breast cancer in Boston, Massachusetts (US) with those in Seoul, South Korea (KR). Materials and Methods: A retrospective review was performed of consecutive patients less than 35 years old with a diagnosis of breast cancer at academic cancer centers in the US and KR from 2000-2005. Patient data were obtained by chart review. Demographic, tumor and treatment characteristics were compared utilizing Pearson's chisquare or Wilcoxon rank-sum tests where appropriate. All differences were assessed as significant at the 0.05 level. Results: 205 patients from the US and 309 from KR were analyzed. Patients in US were more likely to have hormone receptor positive breast cancer, while patients in KR had a higher rate of triple negative lesions. Patients in US had a higher mean body mass index and more often reported use of birth control pills, while those in the KR were less likely to have a sentinel node procedure performed or to receive post mastectomy radiation. Conclusions: Patients under 35 diagnosed with breast cancer in the US and KR differ with respect to demographics, tumor characteristics and management. Although rates of breast conservation and mastectomy were similar, US patients were more likely to receive post mastectomy radiation. The lower use of sentinel node biopsy is explained by the later adoption of the technique in KR. Further evaluation is necessary to evaluate recurrence rates and survival in the setting of differing disease subtypes in these patients.
\end{abstract}

Keywords: Breast cancer - young women - risk factors - management - South Korea - the United States

Asian Pac J Cancer Prev, 16 (6), 2531-2535

\section{Introduction}

Breast cancer is the most common cancer seen in females throughout the world (DeSantis et al., 2014). The epidemiologic profiles of breast cancer in Asian countries differ from Western countries. (Agarwal et al., 2007; Toi et al., 2010). Age-standardized incidence rates of breast cancer are high in North America and western Europe, moderate in South America and eastern Europe, low in Asia (Shibuya et al, 2000). South Korea (KR) along with other Asian countries has in the last decade experienced a more rapid increase in breast cancer incidence than the United States (US) and Europe (Deapen et al., 2002; Suh et al., 2013). Although the overall incidence rate of breast cancer in KR remains lower than that of the US, breast cancer presents at a younger age (Son et al., 2006; Agarwal et al., 2007; Lim et al., 2007; Leong SP et al., 2010; Jung et al., 2013; DeSantis et al., 2014).

Breast cancer in young women (defined as age $<35$ ) has been shown to be more aggressive correlating with lower survival and higher local-regional recurrence (Han et al., 2010; Liukkonen et al., 2011; Han et al., 2011; Kim et al., 2011; Wei et al., 2013).Younger age is an independent predictor in determining adverse outcomes in breast cancer (de la Rochefordiere et al., 1993; Anders et al., 2008). In general, breast cancers diagnosed in younger 
cohorts are more likely to be of larger size, higher-grade, ER/PR negative, have axillary lymph node involvement and exhibit lymphovascular space invasion (Gajdos et al., 2000; Colleoni et al., 2002; Wei et al., 2013; Goksu et al., 2014). A significantly higher incidence in over-expression of HER-2/neu in cancers in women from KR was noted in a study looking at biologic differences between Korean women versus Caucasian women in the US (Choi et al., 2003).

The aim of the current study was to compare the risk factors and management in young women, with breast cancer treated at academic cancer centers in the US and KR.

\section{Materials and Methods}

Institutional databases at Dana Farber/Brigham and Women's Cancer Center in Boston and Asan Medical Center in KR were queried in this retrospective study. After Institutional Review Board approval, all patients less than 35 years old with a diagnosis of invasive breast cancer during the period 2000 to 2005 were included in this study. Data on clinical and patient characteristics, pathological tumor characteristics and treatment history of the patient, were obtained by electronic medical record review.

Triple negative disease was defined as hormone receptors $<1 \%$ positive in both cohorts. Her2/neu status was reported as positive or negative based on IHC of $3+$ and/or FISH testing in both cohorts. Adjuvant therapies available in each country were different during this time period. Survival and local regional failure was not evaluated in this study.

Demographic, tumor and treatment characteristics were compared utilizing Pearson's chi-square or Wilcoxon rank-sum tests where appropriate. All differences were assessed as significant at the 0.05 level.

\section{Results}

Between 2000 and 2005, 205 patients from the US and 309 from KR were available for analysis. Patient demographics are detailed in Table 1. Mean age at diagnosis was 32.1 years in the US and 30.9 years in KR $(\mathrm{p}<0.0001)$. There was no difference in the groups in terms of parity or age at first birth. Patients in the US were more likely to have earlier age of menarche $(\mathrm{p}<0.0001)$, higher body mass index (BMI) $(\mathrm{p}<0.0001)$, use oral contraceptive pills (OCPs) $(\mathrm{p}<0.0001)$, and have a family history of breast cancer $(\mathrm{p}<0.0001)$.

Tumor characteristics are described in Table 2. There was no difference in tumor size, nodal status or incidence of metastatic disease at presentation. Numerically more patients from the US presented with Stage III disease, although this was not statistically significant. Patients

Table 1. Demographics of Patients $<35$ with Breast Cancer in the US and KR

\begin{tabular}{lccc}
\hline & United States $(\%)$ & South Korea $(\%)$ & p-value \\
\hline Mean Age (yrs) & $\mathrm{N}=205$ & $\mathrm{~N}=309$ & $<0.0001$ \\
Mean Age Menarche (yrs) & 32.1 & 30.6 & $<0.0001$ \\
Mean Age First Birth (yrs) & 12.9 & 14.2 & 0.8636 \\
Nulliparity (\%) & 27 & 27.5 & 0.2368 \\
Mean Body Mass Index (kg/m $\left.{ }^{2}\right)$ & $89(43.4)$ & $118(38.2)$ & $<0.0001$ \\
Oral Contraceptive Pill Use (\%) & 25.2 & 22 & $<0.0001$ \\
Reports Family History (\%) & $171(83.8)$ & $23(8.1)$ & $<0.0001$ \\
\hline
\end{tabular}

Table 2. Tumor Characteristics of Patients $<35$ with Breast Cancer in the US and KR

\begin{tabular}{|c|c|c|c|}
\hline & $\begin{array}{l}\text { United States }(\%) \\
\qquad \mathrm{N}=205\end{array}$ & $\begin{array}{l}\text { South Korea }(\%) \\
\qquad N=309\end{array}$ & $\mathrm{p}$-value \\
\hline \multicolumn{4}{|l|}{ Tumor stage } \\
\hline $\mathrm{T} 1$ & $92(45.3)$ & $132(43.4)$ & 0.1221 \\
\hline $\mathrm{T} 2$ & $79(38.9)$ & $142(46.7)$ & \\
\hline $\mathrm{T} 3$ & $23(11.3)$ & $19(6.3)$ & \\
\hline $\mathrm{T} 4$ & $9(4.4)$ & $11(3.6)$ & \\
\hline \multicolumn{4}{|l|}{ Nodal stage } \\
\hline N0 & $97(47.6)$ & $166(53.9)$ & 0.1441 \\
\hline N1 & $77(37.8)$ & $95(30.8)$ & \\
\hline $\mathrm{N} 2$ & $21(10.3)$ & $24(7.8)$ & \\
\hline N3 & $9(4.4)$ & $23(7.5)$ & \\
\hline \multicolumn{4}{|l|}{ Stage } \\
\hline $\mathrm{I}$ & $64(31.2)$ & $96(31.7)$ & 0.2676 \\
\hline II & $88(42.9)$ & $150(49.5)$ & \\
\hline III & $44(21.5)$ & $47(15.5)$ & \\
\hline IV & $9(4.4)$ & $10(3.3)$ & \\
\hline Lymphovascular invasion present & $89(45.6)$ & $109(39.6)$ & 0.1939 \\
\hline Grade 3 Tumor & $121(59)$ & $142(46)$ & 0.033 \\
\hline Hormone receptor positive & 159 (77.6) & $190(61.5)$ & $<0.0001$ \\
\hline HER2 positive & $63(31.3)$ & $84(27.9)$ & 0.4071 \\
\hline Triple negative & $31(15.1)$ & $78(25.2)$ & 0.008 \\
\hline
\end{tabular}

*BCS $=$ Breast conserving surgery; PMRT $=$ Post-mastectomy radiation therapy 

Table 3. Therapy of Patients $<35$ with Breast Cancer in the US and KR

\begin{tabular}{|c|c|c|c|}
\hline & $\begin{array}{l}\text { US (\%) } \\
\mathrm{N}=205\end{array}$ & $\begin{array}{c}\mathrm{KR}(\%) \\
\mathrm{N}=309\end{array}$ & $\mathrm{p}$-value \\
\hline \multicolumn{4}{|l|}{ Systemic therapy } \\
\hline Endocrine therapy & $142(70.0)$ & $170(55.9)$ & 0.0015 \\
\hline Chemotherapy & $188(91.7)$ & $266(86.1)$ & 0.0519 \\
\hline \multicolumn{4}{|l|}{ Local Therapy } \\
\hline \multicolumn{4}{|l|}{ Surgery } \\
\hline $\mathrm{BCS}$ & $88(42.9)$ & $113(36.6)$ & 0.1481 \\
\hline Mastectomy & $108(52.7)$ & $187(60.5)$ & 0.0786 \\
\hline Sentinel node biopsy performed & $114(56)$ & $64(21)$ & $<0.0001$ \\
\hline Radiation after BCS & 87 (99) & $115(94)$ & 0.07 \\
\hline Receiving PMRT by stage & & & $<0.001$ \\
\hline Stage I & $2(7)$ & 0 & 0.15 \\
\hline Stage II & $27(60)$ & $19(19)$ & $<0.0001$ \\
\hline Stage III & $31(91)$ & $35(90)$ & 1 \\
\hline
\end{tabular}

from the US were more likely to have high grade cancers $(\mathrm{p}=0.033)$. Patients from the US were more likely to have hormone positive cancers $(\mathrm{p}<0.0001)$, whereas patients from KR were more likely to have triple negative disease $(p=0.008)$. There was no difference in incidence of HER2 positive cancers or the presence of LVI between US and KR patients.

Local and systemic therapies are detailed in Table 3. There was no difference in receipt of chemotherapy across sites nor in rates of breast conservation with radiation More patients from the US received endocrine therapy. More US patients had sentinel node biopsy performed during the study period. Despite lack of difference in stage at presentation, more US patients received postmastectomy radiation, particularly in the setting of stage II disease $(\mathrm{p}<0.0001)$.

\section{Discussion}

In our study, patients under 35 diagnosed with breast cancer in the US and KR differed with respect to demographics, tumor characteristics and management. Mean age at diagnosis was 32.1 years in the US and 30.9 years in KR, which is consistent with the younger age of presentation in KR. Despite a lower overall incidence of breast cancer $(33.9 / 100,000)$ in $\mathrm{KR}$ when compared to the US (84.7-127.3/100,000) (Jung et al., 2013; De Santis et al., 2014). From 2004 to 2012, the incidence has proportionally increased much more rapidly in KR (Suh et al., 2013). In 2010, the median age at diagnosis in KR was 49, which is higher than previously reported, but still more than 10 years lower than that seen in the US (Ko et al., 2012). Modern series suggests that the median age at diagnosis in KR is gradually shifting toward an older age, similar to that seen in Western countries (Son et al., 2006; Kim et al., 2014). The increasing impact of a Western lifestyle and the rising use of screening mammography may contribute to the observed increases in KR (Ko et al., 2012; Suh et al., 2013). In early 2000, the Korean National Health and Nutrition Examination Survey revealed the rate of mammography was only $22.3 \%$ among women aged $\geq 40$ years in 2001 (Choi et al., 2010). More recently, breast cancer screening rates in KR showed a significant increase from 2004-2012, and screening rates for breast cancer are now approaching 70\% (Suh et al., 2013). When women from Asia immigrate to the United States, their incidence of breast cancer rises rapidly over a short period of time to rates closer to that of their Caucasian counterparts (Deapen et al., 2002).

In our study patients in the US were more likely to have earlier age of menarche, an established risk factor for breast cancer due to increased endogenous estrogen exposure. This variability for age of menarche may also be due to a combination of genetic, ethnic, environmental and nutritional factors (Gaudineau et al., 2010).

Use of OCPs is much more common in the US group than KR group (83.8\% versus $8.1 \%$ ). Many epidemiological studies have investigated the relationship between OCPs use and breast cancer risk; however, the data is conflicting. Some studies have found no association between OCPs use and breast cancer risk (Marhcbanks et al., 2002; Dorjgochoo et al., 2009).

In line with overall rates of obesity in the US versus Korea, we found a higher BMI in the US cohort. There are numerous studies which have investigated the correlation between obesity and breast cancer. The results of those studies showed that obesity may increase risk of breast cancer in postmenopausal women, but no association has been identified for premenopausal women (Huang et al., 1997; van den Brandt et al., 2000; Deng et al., 2001; Reeves et al., 2007; Nelson et al., 2012). A meta-analyses published by Cheraghi et al. showed that an increase in BMI during premenopausal period reduced the risk of breast cancer (Cheraghi et al., 2012). In contrast, Jung and Lee published a meta-analysis of KR women showing that a high BMI for both pre- and postmenopausal groups was shown to have a higher incidence rate of breast cancer (Jung et al., 2009). One possible nutritional effect might be attributed to the KR diet or overall caloric intake.

A positive family history of breast cancer in the US group $(53.6 \%)$ was higher than that of the KR group $(9.7 \%)$. In KR, a family history of breast and/or ovarian cancers was $13 \%$ in one study and BRCA1 and BRCA2 mutations rates in young Korean breast cancer patients were significantly lower than that found by most other studies. 
We found that patients from the US were more likely to have hormone positive cancers, whereas patients from KR were more likely to have triple negative disease. The discrepancy may be due to the difference in the genetic background and epidemiological factors between the two countries. Chuang et al. (2012) investigated whether the prevalence of breast cancer molecular subtypes varied among women of different Asian-American ethnic groups. Although the analysis in that study only included a small number of Korean-American women, the study revealed that Korean-American women had a statistically significant increase in triple negative cancers compared with other Asian groups Korean-American women in the Chuang study were more likely to be younger in age. In addition, young Korean women with breast cancer were reported to have a high prevalence of BRCA 1 and 2 mutations, which were more often associated with ER negative cancers (Chuang et al., 2012).

TNBC is strongly associated with patients of younger age and BRCA1 mutation carriers (Rummel et al., 2013). The KOHBRA study was designed by the Korean Breast Cancer Society to investigate the prevalence of BRCA1/2 mutations in several groups of subjects seen in high-risk breast cancers in the KR population (Han et al., 2011; Son et al., 2012). The results of the KOHBRA study suggest that BRCA1/2 mutations were detected at a higher frequency, particularly in patients with early onset of less than 35 years of age with no family history of breast or ovarian cancer but with other risk factors of genetic disease. Further studies are needed to evaluate the association between genetic and breast cancer cases in young KR women. We also compared local-regional and adjuvant therapies between the two groups. Similar numbers of women received chemotherapy in each group. More women in the US received endocrine therapy, which correlates with the higher number of endocrine-responsive tumors in that population.

In a previous a higher percentage of women with breast cancer underwent mastectomy in KR compared to the US (Leong et al., 2010). Data from our two large academic centers did not show a significant difference in rate of breast conservation versus mastectomy between populations. This may be due to the fact that younger women may be more likely to undergo mastectomy due to fear of local recurrence, genetic predisposition to breast cancer, more advanced disease, family history or occult imaging presentation (Rosenberg et al., 2013). Women in the US were more likely to undergo sentinel node biopsy, which is consistent with prior data, due to the later institutional adoption of the technique in KR (Leong et al., 2010). Similar numbers of young women received radiation after breast conserving surgery, but more women in the US received post-mastectomy radiation therapy. This difference was predominantly seen in the setting of stage II disease where the role for radiation following mastectomy is more controversial. In the US center, features prompting post-mastectomy radiation for Stage II disease include young age, presence of LVI, tumor subtype and lymph node involvement. This study has several limitations including its retrospective nature and a limited data set of two tertiary care centers potentially not necessarily of similar patient catchment. Unlike in KR, the majority of cancer in the US is done in the community setting. In addition we were not able to evaluate for local/ regional/distant failure and overall survival. During the study period adjuvant regimens available and utilized differed between our two institutions. Our study does raise interesting questions on differences and similarities between this age group that warrants further study and validation in larger cohorts in a more modern data set. Also further work is also needed to evaluate the biologic and genomic differences in these populations that contribute to these differing disease characteristics.

In conclusion, our study demonstrates that presenting demographical, pathologic features and management of breast cancer differ between the US and Korean people in women younger than age 35 years. Additional prospective studies in larger series would be required to investigate survival analyses, as well as further genetic background.

\section{References}

Agarwal G, Pradeep PV, Aggarwal V, et al (2007). Spectrum of Breast Cancer in Asian Women. World J Surg, 31, 1031-40.

Anders CK, Hsu DS, Broadwater G, et al (2008). Young age at diagnosis correlates with worse prognosis and defines a subset of breast cancers with shared patterns of gene expression. J Clin Oncol, 26, 3324-30.

Cheraghi Z, Poorolajal J, Hashem T, et al (2012). Effect of body mass index on breast cancer during premenopausal and postmenopausal periods: a meta-analysis. PLoS One, 7, 51446 .

Choi DH, Lee MH, Bale AE, et al (2004). Incidence of BRCA1 and BRCA2 mutations in young Korean breast cancer patients. J Clin Oncol, 22, 1638-45.

Choi DH, Shin DB, Lee MH, et al (2003). A comparison of five immunohistochemical biomarkers and HER-2/neu gene amplification by fluorescence in situ hybridization in white and korean patients with early-onset breast carcinoma. Cancer, 98, 1587-95.

Choi KS, Lee S, Park EC, et al (2010) Comparison of breast cancer screening rates between Korean women in America versus Korea. J Womens Health (Larchmt). 19, 1089-96.

Chuang E, Paul C, Flam A, et al (2012). Breast cancer subtypes in Asian-Americans differ according to Asian ethnic group. J Immigr Minor Health, 14, 754-8.

Collaborative Group on Hormonal Factors in Breast Cancer, (1996). Breast cancer and hormonal contraceptives: collaborative reanalysis of individual data on 53297 women with breast cancer and 100239 women without breast cancer from 54 epidemiological studies. Lancet, 347, 1713-27.

Colleoni M, Rotmensz N, Robertson C, et al (2002). Very young women ( $<35$ years) with operable breast cancer: features of disease at presentation. Ann Oncol, 13, 273-9.

Deapen D, Liu L, Perkins C, et al (2002). Rapidly rising breast cancer incidence rates among Asian-American women. Int J Cancer, 99, 747-50.

de la Rochefordiere A, Asselain B, Campana F, et al (1993) Age as prognostic factor in premenopausal breast carcinoma. Lancet, 341, 1039-43.

DeSantis CE, Lin CC, Mariotto AB, et al (2014). Cancer treatment and survivorship statistics, 2014. CA Cancer $J$ Clin, 64, 252-71.

Dorjgochoo T, Shu XO, Li HL, et al (2006). Use of oral contraceptives, intrauterine devices and tubal sterilization and cancer risk in a large prospective study, from 1996 to 
Young Women with Breast Cancer in the US and South Korea: Comparison of Demographics, Pathology and Management 2006. Int J Cancer, 124, 2442-9.

Foulkes WD, Smith IE, Reis-Filho JS, (2010). Triple-negative breast cancer. $N$ Engl J Med, 363, 1938-48.

Gajdos C, Tartter PI, Bleiweiss IJ, et al (2000). Stage 0 to Stage III Breast Cancer in Young Women. J Am Coll Surg, 190, 523-9.

Gaudineau A, Ehlinger V, Vayssiere C, et al (2010). Factors associated with early menarche: results from the French Health Behaviour in School-aged Children (HBSC) study. BMC Public Health, 30, 175.

Goksu SS, Tastekin D, Arslan D, et al (2014). Clinicopathologic features and molecular subtypes of breast cancer in young women (age $\leq 35$ ). Asian Pac J Cancer Prev, 15, 6665-8.

Han JG, Jiang YD, Zhang CH, et al (2011). Clinicopathologic characteristics and prognosis of young patients with breast cancer. Breast, 20, 370-2.

Han W, Kang SY; Korean Breast Cancer Society (2010). Relationship between age at diagnosis and outcome of premenopausal breast cancer: age less than 35 years is a reasonable cut-off for defining young age-onset breast cancer. Breast Cancer Res Treat, 119, 193-200.

Han SA, Park SK, Ahn SH, et al (2011). The Korean Hereditary Breast Cancer (KOHBRA) study: protocols and interim report. Clin Oncol (R Coll Radiol), 23, 434-41.

Huang Z, Hankinson SE, Colditz GA, et al (1997). Dual effects of weight and weight gain on breast cancer risk. JAMA, 278, 1407-11.

Jung D, Lee SM (2009). BMI and breast cancer in Korean Women: a meta-analysis. Asian Nurs Res, 3, 31-40.

Jung KW, Won YJ, Kong HJ, et al (2013). Prediction of cancer incidence and mortality in Korea, 2013. Cancer Res Treat, 45, 15-21.

Kahlenborn C, Modugno F, Potter DM, et al (2006). Oral contraceptive use as a risk factor for premenopausal breast cancer: a meta-analysis. Mayo Clin Proc, 81, 1290-302.

Kim IK, Park S, Hwang H, et al (2011). Clinical significance of age at the time of diagnosis among young breast cancer patients. J Breast Cancer, 14, 314-21.

Kim Z, Min SY, Yoon CS, et al (2014). The basic facts of korean breast cancer in 2011: results of a nationwide survey and breast cancer registry database. J Breast Cancer, 17, 99-106.

Ko, BS, Noh WC, Kang SS, et al (2012). Changing patterns in the clinical characteristics of Korean breast cancer from 19962010 using an online nationwide breast cancer database. $J$ Breast Cancer, 15, 393-400.

Lamartiniere CA, Cotroneo MS, Fritz WA, et al (2002). Genistein chemoprevention: timing and mechanisms of action in murine mammary and prostate. J Nutr, 132, 552-558.

Leong SP, Shen ZZ, Liu TJ, et al (2010). Is breast cancer the same disease in asian and western countries? World J Surg, 34, 2308-24.

Lim SE, Back M, Quek E, (2007). Clinical observations from a breast cancer registry in asian women.World J Surg, 31, 1387-92.

Liukkonen S, Leidenius M, Saarto T, et al (2011). Breast cancer in very young women. Eur J Surg Oncol, 37, 1030-7.

Marchbanks PA, McDonald JA, Wilson HG, et al (2002). Oral contraceptives and the risk of breast cancer. $N$ Engl J Med, 346, 2025-32.

Nelson HD, Zakher B, Cantor A, et al (2012). Risk factors for breast cancer for women aged 40 to 49 years: a systematic review and meta-analysis. Ann Intern Med, 156, 635-48.

Reeves GK, Pirie K, Beral V, et al (2007). Cancer incidence and mortality in relation to body mass index in the Million Women Study: cohort study. BMJ, 335, 1134 .

Rosenberg SM, Sepucha K, Ruddy KJ. Choosing mastectomy over lumpectomy: Factors associated with surgical decisions 